\title{
Treatment of enthesopathies with peritendinous injection of oxygen/ozone and ultrasound-guided collagenotherapy
}

\author{
Roberto Brembilla \\ Department of Radiology, Treviglio Hospital, Treviglio (BG), Italy
}

\begin{abstract}
Our own experience about a new way of therapy for ligament e tendon injuries, degenerative or traumatic with use of double treatment: oxygen-ozone injection and collagen injection in the site of the pathology with ultrasound guide.
\end{abstract}

\section{Introduction}

Treatment of tendon and ligamentous structures is extremely difficult and does not guarantee results. In recent years as part of the physiatrist, rehabilitative and orthopedic medicine, numerous technical dependent by equipment were introduced (physical therapies at start, then shock waves, and recently the Tecar therapy), all of them, if well applied and following an adequate patient selection, may have positive results. In some cases, forcing the indication, tissue suffering is recorded, mostly caused by shock waves that produce a locoregional hyperemia.

Aim of this work is to define a therapeutic method easy to use, not harmful, effective and reproducible.

The power of oxigenozone for edema tissue is well known.

Instead, the power of synthetic collagen for restoring tendon and ligament integrity is not known.

Low cost, high tolerance, no risk of doping are the high spots of this new way of treatment.

We put our attention on the health of the subject, wheseas instead restitution ad integrum was not the most urgent.

Correspondence: Roberto Brembilla, Department of Radiology, Treviglio Hospital, P.le Ospedale n. 1 - 24047 Treviglio (BG), Italy.

E-mail: roberto.brembilla@ospedale.treviglio.bg.it

Key words: Oxygenozone; Collagenotherapy; Enthesopathies; Ultrasound.

Received for publication: 23 June 2016.

Accepted for publication: 18 July 2016.

(C) Copyright R. Brembilla, 2016

Licensee PAGEPress, Italy

Ozone Therapy 2016; 1:6273

doi:10.4081/ozone.2016.6273

This article is distributed under the terms of the Creative Commons Attribution Noncommercial License (by-nc 4.0) which permits any noncommercial use, distribution, and reproduction in any medium, provided the original author(s) and source are credited.

\section{Materials and Methods}

Between January 2009 and January 2013269 patients with tendonligament disease noted and documented were identified, divided into two big groups: i) 140 patients (81 men) carrying traumatic pathology not completely fractured, excluded from the surgery act; mean age 28 years; ii) 129 patients (78 women) carrying degenerative pathology; mean age 47 years.

\section{Group A}

For 66 patients, as part of the traumatic pathology, the most important part is the tendon-muscular compartment of the quadriceps and hamstring and of the leg flexors, for practicing football. ${ }^{1}$

Thirteen patients suffered from the Achilles tendon pathology for repeated microtrauma, mainly runners.

Five patients suffered from the iliotibial band syndrome.

Five patient presented tendinopathy of the goosefoot.

Six suffered from enthesitis epicondyle-epitrochlear.

Twelve presented tendonitis of the rotator cuff and of the humeral biceps long tendon.

Eight patients presented inflammation of the ankle ligament-capsular.

Three patients had symptoms at the first finger abductor tendon and its associated carpal ligament.

Three patients had elective soreness at the origin of the longus tendon and the medial triceps.

Three patients had pain and tendon deficiency at the extensor in the distal interphalangeal joint at the fingers after distractive trauma.

Fourteen manifested signs of adductors insertional enthesitis isolated or inserted within a groin.

Two cases of fasciitis of the anterior rectus muscles.

\section{Group B}

Forty-five patients presented subacromial shoulder conflict with or without the SubAcromion deltoid bursitis, all of them with tenosynovitis at the humeral tendon of the long head of the brachial biceps. The $65 \%$ of those presented calcifications both intra and extra tendon with variable dimensions between 3 and $21 \mathrm{~mm}$. In these cases a therapy by assumption of colchicine CF, two a day for a month, one a day for two months, exploiting the chelating effect of calcium in also proposed.

Twelve patients had trochanteric bursitis and enthesopathy at the fascia lata.

Fourteen patients presented epicondyle-trochlear syndrome, in the latter case also with syndrome of the ulnar tunnel -80\%.

Eighteen patients experienced forefoot syndromes like neuroma or intermetatarsal bursitis.

There were 9 case of enthesopathy of the Achilles tendon, with significantly impaired function, granulomatous infiltrating accertained by ultrasound and proceedings of therapies without success. 
Sixteen cases manifested capsular-ligament complications after rizoarthrosis.

Eight cases involved an insertion of the patellar ligament on the anterior apofisi tibialis -0sgood-Schlatter; mean age 12 years.

A case of juvenile malleolus apophysitis; mean age 9 years.

Six cases of post-fracture or degenerative capsulitis at the ankle with or without peroneals tendinopatia.

\section{Technique}

Patients were investigated at first with ultrasound and /or magnetic resonance (MR), those positive to tendon pathology underwent further own personal ultrasound pretreatment. Needles from 27 to $30 \mathrm{G}$ were used to the treatment site, with length varying from 4 up to $20 \mathrm{~mm} .^{2}$ The injection procedure was performed with ultrasound assistance for more precision in the treatment that has to be targeted. The procedure is divided into two moments. Injection of a quantity that can vary from 2 and 6-8 cc of oxygen-ozone $\left(\mathrm{O}_{2}-\mathrm{O}_{3}\right)$ at $5 \mathrm{mc} / \mathrm{mL}$ in dual or triple injection depending on the body part.1,3

Then injection of 2-3 mL of liquid collagen with a single procedure in the focal site of the pathology shown by ultrasonography, clinical symptoms and functional deficits.

The author is a supporter of once-weekly treatment since this is a natural method that goes to stimulate a physiological repair process, thus requiring its recovery time. None of the patients were treated with steroid because some patients were professional athlets and for the long acting damage caused by steroids on tendons and ligaments. Also by the association of the $\mathrm{O}_{2}-\mathrm{O}_{3}$ therapy with collagen we can obtain effect: i) anti edema; ii) inflammation typical $\mathrm{O}_{2}-\mathrm{O}_{3}$; iii) nutritive; iv) replenishing of collagen, key element of connective tissues derived.

The ultrasound guidance with variable frequency linear probe allows to identify with certainty the site of pathology. In some cases only $\mathrm{O}_{2}-\mathrm{O}_{3}$ was injected in the intra-articular zone for adhesive capsulitis of the shoulder, carpometacarpal osteoarthritis, ankle capsulitis of preferentially tibialis anterior or extemal submalleolar. ${ }^{4}$

It is important to notice the absence of complications and pain at the moment of the collagen injection: it is advisable, therefore, to create small wheals in pathology headquarters, which are absorbed within about 12 hours, resulting in a therapeutic effect which reaches its maximum expression after 3-4 days. The number of sessions is variable and depends basically on two facto rs: the body part and the duration of the pathology. A cycle varies between 6 and 12 sessions. In the longer cycles the latest fortnightly sessions are proposed not to create dependency of the hole.

\section{Results}

The healing with remission of the symptom is $100 \%$ in the traumatic sports pathology.

The optimal clinical result in the degenerative pathology is $90 \%$; acceptable result in the remaining $10 \%$. The most resistant to therapy are, obviously, the Achilles tendon and the enthesopathic elbow districts, also because most of the people approach our therapy belatedly and after numerous therapeutic failures. The Achilles tendon in particular, after the recover of the pain, remains slightly thickened but the functionality is recovered.

Our patients with Achilles pathology were professional athletes: they obtained good results in medium-, long-term, depending on the severity of injury (four/five months).

Notable is the recovery of the shoulder calcifications: personally I disagree with the use of the shock wave, for the long-term damage created at the local level and the failure dose. I prefer to create a physiological environment of tendon edema reabsorption, to make the chelat- ing effect of colchicine, to introduce a Fisiokinesy therapy cycle and later motor activity tended to oxygenate the treated muscle-tendon district.

\section{Conclusions}

The use of the double therapeutic approach, $\mathrm{O}_{2}-\mathrm{O}_{3}$ and collagen therapy, opens new perspectives of treatment: i) the patient is followed by the doctor from diagnosis to therapy and until the healing: this allows the doctor to follow the evolution, even with greater patient satisfaction. Any other therapeutic figures play a rehabilitative and complementary function, aimed at a more rapid functional recovery; ii) the absolute harmlessness of the treatments performed makes the method also advisable to professional athlets, for once free from the pharmacological nightmare; iii) the high clinical response allows ozonotherapeutics to recover confidence in their means and to reconnect all of that minor pathology that until now they tended to send to others. Therefore they create new areas, with very low amount of risk and high cost-benefit; iv) the results are very encouraging with a clinical response of $100 \%$ in all treated, both the group A and the group B. The best response is obtained in young, athletic subjects who need a speedy recovery. Recovery time is halved compared with normal medical-sports practice and the response to the tendon repair is more effective, with a reduction in post trauma fibrosis phenomena that affect the athlete, fearful of recurrence in the same muscle site. The association of the therapeutic combination of $\mathrm{O}_{2}-\mathrm{O}_{3}$ collagen, can be also supported by Tecar therapy, enhancing even further the therapeutic efficacy and reducing the forced stop, with greater satisfaction, particularly of the professional athlete. However, also in subjects with degenerative pathology the therapeutic response is $100 \%$, with optimal response of $90 \%$ and acceptable in $10 \%$ of cases in this regard, the lag time between the onset of pathology and the therapy is decisive: the faster the action, the more favorable is the therapeutic course and valid the answer. In elderly patients a pathogenic noxa, if not resolved quickly, tends to create a chronic pathology, triggering more vicious circles fundamentally linked to the joint disability: edema or neuro-myalgia not to use are the biggest enemies of the recovery. It can therefore be assessed case by case, the introduction of other rehabilitation practices, e.g. lymphatic drainage massage, physiokinesitherapy, motor rehabilitation in water etc. It is also crucial to stimulate the patient to constantly introduce, where possible, an active motor activity and not just passive rehabilitation. It was also noted that, while the patient of the group A closes its process with the restitutio ad integrum, the patient of the group B undergoes spontaneously fortnightly or monthly or quarterly calls, depending on the clinical indication but also the physiological need, and loyalty to the doctor-patient relationship; v) the absolute collagen tolerability allows to treat even atopic or with severe systemic disease subjects who, conversely, cannot bear the traditional drug or manual therapies.

\section{References}

1. Gjonovich A, Marchetto R, Montemara E, Girotto T. Refractoary tendinopathies of the knee: use of oxigen-ozone therapy. Riv It Ossigeno-Ozonoterapia 2003;2:187-92.

2. Benvenuti P. Oxygen-ozone treatment of the knee, shoulder and hip. A personal experience. Riv It Ossigeno-0zonoterapia 2006;5:135-44.

3. Lin $\mathrm{Q}$, Chen $\mathrm{H}$, Lu C, et al. Effects of ozone on sciatic nerve in rat. Interv Neuroradiol 2011;17:281-5.

4. McNally EG, Shetty S. Plantar fascia: imaging diagnosis and guided treatment. Semin Musculoskelet Radiol 2010;14:334-43. 